\title{
The Ionization and Expansion of a Globule of Interstellar Gas overun by a Weak R Type Ionization Front
}

\author{
J. S. Berry \\ School of Physical Sciences, The New University of Ulster, Coleraine, Co. Londonderry, \\ North Ireland
}

(Z. Naturforsch. 32 a, 692-696 [1977] ; received April 26, 1977)

\begin{abstract}
The flow of the ionized gas behind a contracting ionization front is investigated for spherical symmetry. A similarity solution is given when the initial density distribution in the neutral hydrogen is $\omega_{0} / r^{\alpha}$ where $r$ is the distance from the centre of contraction.
\end{abstract}

\section{Introduction}

Several authors ${ }^{1-4}$ have, in recent years, studied the effect on a globule of interstellar gas (neutral hydrogen) when subject to ionizing radiation from outside.

The radiation causes the hydrogen to be ionized; the transition between the ionized and non-ionized regions is called an ionization front. This ionization front is called R-type or D-type if its velocity relative to that of the neutral gas ahead is either supersonic or subsonic respectively. Further the terms 'weak R' and 'strong D' or 'weak D' are used according to whether the ionization front travels supersonically or subsonically relative to the gas behind the front respectively.

Dyson ${ }^{1}$ assumed a critical D-type ionization front and an isothermal gas sphere and thus his model did not allow the ionization front to evolve through the weak $\mathrm{R}$, strong $\mathrm{D}$ and weak $\mathrm{D}$ phases. The present author, in a previous paper, Berry ${ }^{3}$ gave a numerical solution for the gas flow in an HII region behind an ionization front contracting in a globule of neutral hydrogen initially in isothermal, gravitational equilibrium. The model allowed for the ionization front to evolve to a weak D-type with a strong shock front travelling ahead. The reflection of this shock front at the centre of symmetry and the ultimate interaction with the ionization front was not considered. This latter problem was investigated by Tenorio-Tagle ${ }^{4}$. The globule of neutral hydrogen was taken to have constant density and various weak R-type ionization fronts (produced by converging or by a plane parallel radiation field) incident on a spherically symmetric globule were considered. The computations suggest that, due to the shock/ioniza-

Reprint requests to Dr. J. S. Berry, The New University of Ulster, Coleraine, Co. Londonderry, N. Ireland. tion front interactions, high density globules of neutral hydrogen can exist within an HII region. However for lower density cases, the globule was quickly overrun by the weak R-type front before it could fully evolve. This paper investigates such a problem and the subsequent expansion of the HII region by seeking a similarity solution.

In this paper a mathematical model is taken such that no length scale is involved, thus enabling a similarity-type solution to be sought. The advantage of such a solution allows a discussion of the various governing parameters to be made. Accordingly it is assumed that the initial density distribution of the neutral gas forming the cloud is of the form $\omega_{0} / r^{\alpha}$ where $\omega_{0}$ and $\alpha$ are constants and $r$ is the distance from the centre of symmetry. If recombinations and other cooling mechanisms are taken into account the temperature of the ionized gas may be assumed uniform. For a fuller discussion of this see Goldsworthy ${ }^{6}$.

Two phases of the gas motion are considered :that of the ionized gas behind the contracting ionization front and that of the gas after the ionization front has reached the centre of symmetry. These are called the "contraction phase" and "expansion phase" respectively. The analysis closely follows that of Guderley ${ }^{5}$ who gave a similarity solution to the problem of a contracting shock wave. In the contraction phase it is assumed that $t<0$ and in the expansion phase $t>0$.

It is found that with the similarity variable chosen a similarity solution can only be found for weak R-type ionization fronts. The solutions are used to suggest a method of estimating the age of fully ionized gas clouds overrun by very fast moving weak R-type ionization fronts. The similarity solution does require severe restrictions on the physical model taken, these restrictions are discussed in Sec- 
tion 4; however the analysis is mathematically simple, and will act as a guide to what might happen in any given physical configuration.

\section{Equations of Motion in the HII Region}

Goldsworthy ${ }^{6}$ has fully discussed the equations of motion and the processes leading to isothermal conditions in the ionized HII region. Denoting the sound speed by $c$, which is uniform within the HII region the appropriate similarity variable to be used is $\eta=c t / r$. The similarity forms for the flow variables in the HII region are

$$
\begin{array}{ll}
\text { velocity, } & u=(r / t) U(\eta), \\
\text { density, } & \varrho=\left(1 / r^{\alpha}\right) w(\eta), \\
\text { pressure, } & p=c^{2} \varrho,
\end{array}
$$

and the equations of motion for $u$ und $\varrho$ become

$$
\begin{aligned}
& \eta \frac{\mathrm{d} U}{\mathrm{~d} \eta}=\frac{U(1-U)^{2}+\eta^{2}(\alpha-3 U)}{(1-U)^{2}-\eta^{2}}, \\
& \frac{\eta}{w} \frac{\mathrm{d} w}{\mathrm{~d} \eta}=\frac{(\alpha-2) U(1-U)+\eta^{2}}{(1-U)^{2}-\eta^{2}},
\end{aligned}
$$

\section{The Solution for weak R-type Fronts}

\subsection{Contraction Phase}

As a consequence of the similarity assumption the weak R-type ionization front travels into the neutral gas with constant velocity, $u_{f}$, so that its position at time $t(-\infty<t<0)$ is

$$
r_{f}=-u_{f} t
$$

In the analysis which follows $u_{f}$ will be taken as a given parameter, the value of which depends on the radiative output of the distribution of stars at infinity surrounding the globule. Across the ionization front the following relations hold

$$
\begin{aligned}
\eta_{f}^{2} & =U_{i}\left(1-U_{i}\right), \\
w_{i} & =w_{\eta} /\left(1-U_{i}\right),
\end{aligned}
$$

where $\eta_{f}$ is the value of $\eta\left(=-c / u_{f}\right)$ at the ionization front and $u_{i} / c=U_{i} / \eta_{f}$ is the gas velocity on the ionized side of the front ${ }^{6}$.

Equations (1) and (2) are solved numerically using the Runge-Kutta-Merton method. The form of the solution to (1) near the origin $(\eta=0, r=\infty)$ is

$$
U \approx q \eta+\alpha \eta^{2}
$$

where, since $u / c=U / \eta, q$ is the non-dimensional gas velocity $\left(u_{\infty} / c\right)$ at infinity. The following simple analysis shows that, for $u_{f / c}$ sufficiently large, if $\alpha>1, q>0$ and if $\alpha<1, q<0$ :

Suppose that the solution to Eq. (1) be approximately given by (5) everywhere in the ionized gaseous region. This, since it applies for small $\eta$, will correspond to faster moving fronts, e.g. $\eta$ varies from 0 to 0.1 when a front speed of $10 \mathrm{c}$ is taken. At the ionization front $U$ and $\eta$ satisfy $U(1-U)=\eta^{2}$ so that on substituting $U$ from (5) we have

$$
\eta_{f}=-1 /\left(u_{f / c}\right)=q /\left(1-\alpha+q^{2}\right),
$$

from which it is seen that, since for contraction $t<0$, giving $\eta_{f}<0$,

$$
\alpha<1 \text { implies } q<0 \text { i. e. inflow }
$$

and $a>1$ implies $q>0$ i. e. outflow.

Thus $\alpha=1$ is a critical value at which the gas speed at infinity is zero.

The integral curves of Eq. (1) through the origin, for $\alpha=2$ and different values of $q$, in the $\eta<0$ half plane are shown in Figure 1. The ionization front transition relation (3) is labelled "Rif" curve in Figure 1. The line $|1-U|=|\eta|$ separates regions where the speed of the ionization front relative to the flow behind the front is supersonic or subsonic. A weak R-type front is such that $\left|u_{f}-u_{i}\right|>c$ so that

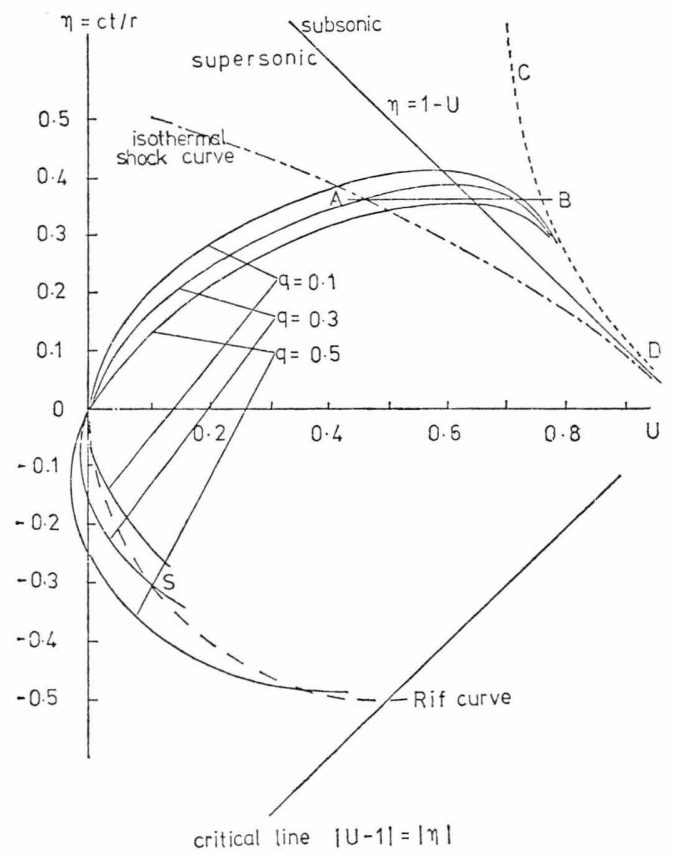

Fig. 1. The $(U, \eta)$ plane representing the solution of differential Eq. (1) for $\alpha=2$. 
we look for an integral curve which intersects the "Rif" curve on the supersonic side of the critical line. The integral curve SO represents a typical solution for a weak R-type front. S represents the ionization front, the speed of which can be found from the value of $\eta$ at the point $\mathrm{S}$ i. e. $u_{f} / c=1 / \eta_{s}$; and the gas velocity behind the front is found from the values of $U$ and $\eta$ at $S$ i. e. $u_{i} / c=U_{s} / \eta_{s}$. Figure 2 shows the variation of $q\left(=u_{\infty} / c\right)$ and $u_{i} / c$ with the front speed $u_{f} / c$; the gas velocity in the HII region is bounded by these two values. The dotted line in Fig. 2 represents Eq. (6), from which it can be seen that Eq. (5) and the subsequent argument holds when $u_{f}>6 c$.

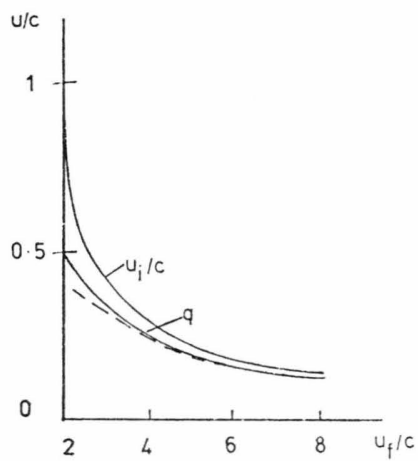

Fig. 2. The variation of $u_{i} / c$ and $q\left(u_{\infty} / c\right)$ with $u_{f} / c$ for a weak $\mathrm{R}$ type ionization front and $\alpha=2$. The dashed curve for $q$ represents the approximate solution given by Equation (6).

\subsection{Expansion Phase}

When the ionization front reaches the centre of the condensation the HII region has density and velocity distributions $\varrho=\Omega / r^{\alpha}$ and $u / c=q$, respectively, where

$$
\frac{\Omega}{w_{0}}=\frac{1}{1-U_{i}}=\frac{1}{\frac{1}{2}+\frac{1}{2}\left[1-4 /\left(u_{f / c}\right)^{2}\right]^{\frac{1}{2}}} .
$$

Thus $\Omega / \omega_{0} \rightarrow 1$ and $q \rightarrow 0$ as $u_{f} / c \rightarrow \infty$; so that the greatest change in density and velocity will be for smaller values of $u_{f} / c$. (For weak R-type fronts $u_{f} / c \geqq 2$ giving $\Omega / \omega_{0} \leqq 2$.) This suggests that even higher speeds may be possible in the HII region if we have weak D or strong D-type ionization fronts.

The flow in the now fully ionized hydrogen globule is found by integrating Eq. (1) and (2) with $\eta>0(t>0$ for expansion) and $\alpha=2$. The boundary condition at the centre $(r=0)$ is $u=0$. At $r=\infty(\eta=0)$ the required condition is $u / c=q$. Now to satisfy these two conditions it is necessary to consider two regions separated by an expanding isothermal shock front $\left[r=r_{s}(t)=u_{s} t, t>0\right]$. The assumption made here is that there is sufficient radiation present to keep the globule fully ionized during the expansion phase, and that a recombination front does not follow the isothermal shock.

For the 'centre side region' $0<r<r_{s}\left(\infty>\eta>\eta_{s}\right)$ Eq. (1) has asymptotic solution $U \rightarrow \frac{2}{3}, \eta \rightarrow \infty$ and CBD represents the integral curve of (1) in this region. Across an isothermal shock the non-dimensional velocity $U$ and the similarity variable $\eta$ satisfy the following relation

$$
U_{+}=1-\eta^{2} /\left(1-U_{-}\right)
$$

where $U_{+}$is the value of $U$ on the "infinity side" of the shock $\left(r>r_{s}\right)$ and $U_{-}$is the value of $U$ on the "centre side" of the shock $\left(r<r_{s}\right)$. In Fig. 1 the "isothermal shock curve" represents transitions across the shock from a point $\left(U_{-}, \eta_{s}\right)$ to a point $\left(U_{+}, \eta_{s}\right)$. The integral curves of (1) on the "infinity-side" region $r_{s}<r$ are those curves which radiate from the origin 0 for different values of $q$.

A typical solution for the flow in the fully-ionized gas cloud is given by $\mathrm{OA}, \mathrm{BC}$ where $\mathrm{AB}$ represents the transition across the isothermal shock. The shock speed is given by $1 / \eta_{A}$ and the gas speeds on either side of the shock are given by $U_{A} / \eta_{A}$ and $U_{B} / \eta_{A}$.

Figure 3 shows how the speed of the expanding isothermal shock in the expansion phase depends on the speed of the contracting ionization front in the contraction phase for $\alpha=2$. The strength of the shock depends on the velocity and density distributions left by the contracting ionization front. In

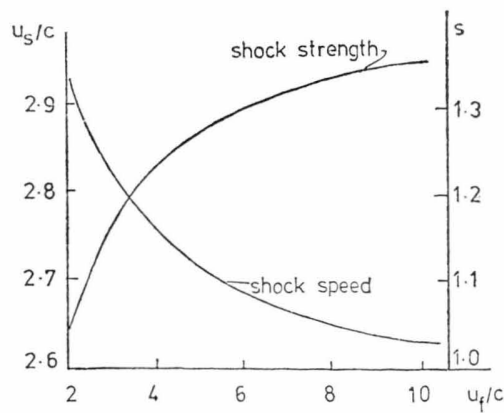

Fig. 3. Diagram showing how the speed and strength of the expanding isothermal shock in the expansion phase depends on the speed of the contracting ionization front in the contraction phase. 
terms of $U_{+}$and $U_{-}$the shock strength $s$ is given by

$$
s=\left(U_{-}-U_{+}\right) /\left(1-U_{-}\right) .
$$

The variation of $s$ with $\left(u_{f} / c\right)$ is also shown in Figure 3.

For other values of $\alpha$ the asymptotic solution for large $\eta$ is $U=\alpha / 3$ and each integral curve CBD passes through the point $U=1, \eta=0$. If $\alpha>3$ then $U \geqq 1$ for all values of $\eta$ so that the "isothermal shock curve" is in the region $U \geqq 1, \eta \geqq 0$. Thus since the integral curves of Eq. (1) lie in the region $U \leqq 1, \eta \geqq 0$ the only point of intersection between these curves and the "shock curve" is the point $U=1, \eta=0$. This solution would represent a shock which has moved instantaneously to infinity.

Figure 4 shows how the density distribution in the fully ionized globule varies with the distance from the centre of the globule. This curve is drawn for one value of $q$ and is obtained by integrating Equation (2).

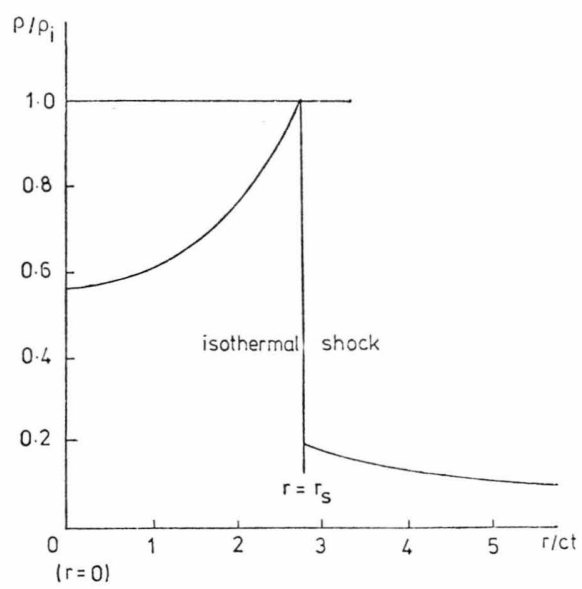

Fig. 4. The density distribution in the fully ionized gas cloud for $\alpha=2$.

\section{Discussion and Conclusions}

To fit a similarity solution to the problem of an ionization front contracting into a globule of neutral hydrogen of initial density distribution $\omega_{0} / r^{\alpha}$, it has been found necessary to introduce a constant velocity at infinity; a velocity which corresponds to an inflow for $\alpha<1$ and an outflow for $\alpha>1$, it being zero for $\alpha=1$.

If required, a condition $u=0$ at infinity could be applied by fitting a shock wave in the HII region when there is a gas outflow $(\alpha>1)$ and a rarefaction wave when there is a gas inflow $(\alpha<1)$. The magnitude of the gas velocity in the HII region varies from approximately $12 \mathrm{~km} / \mathrm{sec}$ just behind the ionization front to $6 \mathrm{~km} / \mathrm{sec}$ at large distances from the centre of symmetry for a slower moving weak R-type front with speed $25 \mathrm{~km} / \mathrm{sec}(2 \mathrm{c})$ and from approximately $2.6 \mathrm{~km} / \mathrm{sec}$ to $2.4 \mathrm{~km} / \mathrm{sec}$ for a front moving with speed $61 \mathrm{~km} / \mathrm{sec}(5 \mathrm{c})$. These values are given for the case $\alpha=2$. The magnitude of the gas velocity in the HII region is comparable with that in the numerical solution, Berry ${ }^{3}$.

For the expansion phase it is found that $\alpha=3$ is the critical value for the existence of a shock front expanding with finite speed. For $\alpha>3$ the shock travels to infinity instantaneously, whereas for $\alpha<3$ the shock moves out from the centre of expansion $r=0$ at a finite speed. The reason is simply that for $\alpha<3$ there is infinite mass at infinity whereas for $\alpha>3$ there is infinite mass at the centre representing a source of infinite energy.

D-type ionization fronts have not been considered in this paper. A D-type ionization front is preceded by a contracting strong shock wave. Work has been done by Guderley ${ }^{5}$, and others, on the similarity solution of a contracting shock wave, the gas density distribution being of the form $\varrho=\Omega(\eta)$ where $\eta=t / r^{\lambda}$. It was found that in order to fit a contracting strong shock for $t<0$ so that a reflected shock was obtained for $t>0, \lambda=1 / 0.717$. With the similarity variable $\eta=c t / r$ only a particular value of $\alpha$ in the initial density distribution $\omega_{0} / r^{\alpha}$ will yield D-type ionization fronts.

The full solution, including the expansion phase, can be used to estimate the age of fully ionized gas globules which have been subjected to ionizing radiation from outside. The observed density distribution in the globule will give the speed of the expanding shock and Fig. 3 will give an approximate speed of the ionization front which exists during the contraction phase. If the radius of the globule is $r_{*}$ and the expanding shock is distance $\boldsymbol{r}_{s}$ from the centre, then an estimate of the age of the globule would be $r_{*} / u_{f}+r_{s} / u_{s}$, with a small correction needed if the globule has expanded in radius from its initial value. In fact if $u_{f}$ is large this expansion will be negligible since gas velocities in the HII region are small.

Finally it must be emphasized that the similarity solution can serve only as a guide to what might happen in any given physical configuration. It does initially require a neutral gas cloud of infinite ra- 
dius to exist and that ionizing radiation is incident on it for all time. This is somewhat unphysical since in reality the globule would be of finite radius and would be overtaken by an ionization front moving through the surrounding, less dense, medium. Obviously spherical symmetry would not apply.

1 J. E. Dyson, Astrophys. Space Sci. 2, 461 [1968].

2 J. E. Dyson, Astron. Astrophys. 27, 459 [1973].

3 J. S. Berry, Astron. Astrophys. 44, 401 [1975].

4 G. Tenorio-Tagle, Astron. Astrophys. 54, 517 [1977].

\section{Acknowledgements}

This work was performed during the tenure of an S.R.C. research grant at the University of Leeds, under the supervision of Professor F. A. Goldsworthy, to whom I express my thanks for his advice and interest.

5 G. Guderley, Luftfahrtforschung 19, 302 [1942].

6 F. A. Goldsworthy, Phil. Trans. Roy. Soc. A 253, 277 [1961]. 